\title{
Die Berufung Karl Gustav Jungs (1794-1864) nach Basel und ihre Vorgeschichte
}

Von Huldrych M. Koelbing

In seiner Studie über «Alexander von Humboldt und Frankreich» hebt Heinz Balmer als besondere Qualität jenes großen Geistes seine Fähigkeit zur «Auffindung besonderer Begabungen» hervor ${ }^{1}$. Zu den jungen Leuten, die Humboldt in Paris entdeckte und denen er durch seine Fürsprache ein ihnen angemessenes Wirkungsfeld erschloß, gehört der aus Mannheim gebürtige Dr. med. Karl Gustav Jung (1794-1864). Humboldt hat Jung nach Basel gewiesen, wo zur Zeit seiner Wahl auf den gesetzlichen Lehrstuhl der Chirurgie, Anatomie und Geburtshilfe die medizinische Fakultät kläglich dahinserbelte. Als ungemein vielseitiger Dozent, Organisator und Animator gab Jung ihr Impulse zu einem neuen Aufschwung. Als die Trennung des Kantons Basel und die damit verbundene Teilung des Staatsvermögens 1833 die Existenz der ganzen Universität in Frage stellte, war es für Jung wie für die meisten seiner Kollegen eine Selbstverständlichkeit, ihrer Hochschule trotz halbierter Besoldung die Treue zu halten. Von 1850 bis 1855 vertrat Karl Gustav Jung die innere Medizin - die sein eigentliches Fach war - sogar als unbesoldeter Ordinarius. Ein klinisch-internistischer Unterricht nach modernen Vorstellungen begann in Basel (im Gegensatz zu Zürich und Bern) freilich erst nach Jungs Tod aufgrund des sog. «Klinikenvertrags» (1865), der das Bürgerspital zum Universitätsspital machte, und mit der Berufung Karl Liebermeisters (1833-1901) aus Tübingen zum Ordinarius und Chefarzt der neugeschaffenen medizinischen Universitätsklinik.

Karl Gustav Jungs Vater Franz Ignaz Jung (1759-1831) war Arzt in Mannheim; seine Mutter Maria Josepha geb. Ziegler war 1770 geboren. Bis in unsere Zeit hinein hielt sich das Gerücht, Karl Gustavs wirklicher Vater sei Johann Wolfgang Goethe gewesen. Als ich seinen Enkel Carl Gustav Jung (1875-1961) in Küsnacht bei einem Besuch am 6. November 1954 auf das m. E. unhaltbare Gerücht hin ansprach, nahm er nicht direkt dazu Stellung, sondern sagte: Seine Urgroßmutter Maria Josepha Jung sei, nach den erhaltenen Briefen zu schließen, eine geistig bedeutende Frau gewesen. Ihre Ehe sei unglücklich gewesen. Handschrift und Inhalt der Briefe ließen es nicht als ausgeschlossen erscheinen, daß an der Überlieferung einer Liebesbeziehung zu Goethe etwas sein könnte. In Tat und Wahrheit gibt jedoch Goethes Biographie nicht den geringsten Anhaltspunkt für diese Vermutung $\mathbf{a b}^{2}$. 
Nach 3 1/2 jährigem Studium wurde Karl Gustav Jung 1816, 22 jährig, in Heidelberg summa cum laude zum Doktor der Medizin promoviert. Im folgenden Jahre unternahm er eine größere Studienreise und besuchte verschiedene Universitätsstädte in Mitteldeutschland, unter anderen Jena. Es traf sich wohl nicht zufällig, daß er gerade zur Zeit des großen Wartburgfestes vom 18. Oktober 1817 dort weilte, jenes doppelten Gedenktages für Luthers Thesen-Anschlag (1517) und den Leipziger Sieg über Napoleon (1813), an dem Studenten aus ganz Deutschland ihrem Streben nach einem freiheitlich geeinten Vaterland laut und stürmisch Ausdruck verliehen. Unter den Burschenschaftern, mit denen Jung sich in Jena anfreundete, waren der Theologiestudent Karl Ludwig Sand und die Brüder August und Karl Follen, denen auch gewaltsame Mittel zur Erreichung ihres guten Zweckes erlaubt schienen; Jung selber war ebenfalls für Freiheit und Recht begeistert, aber eine durchaus friedliche Natur. Von den Jenenser Professoren war es kein Mediziner, sondern der Philosoph Jakob Fries (1773 bis 1843), der auf Jungs Denken nachhaltigen Einfluß gewann; er stellte die persönliche Würde in den Mittelpunkt seiner Ethik und forderte demgemäß liberale Reformen im Staat. Jung reiste weiter nach Berlin und kam (Ende 1817 oder Anfang 1818) in das Haus des Buchhändlers Georg Andreas Reimer. Dort versammelte sich jeden Donnerstag ein Kreis geistig regsamer, liberal gesinnter Männer. Unter ihnen traf Jung zwei Theologen: Wilhelm Martin Leberecht de Wette (1780-1849), seinen späteren Kollegen an der Universität Basel, und den großen Schleiermacher (Friedrich Ernst Daniel Schleiermacher, 1768-1834). Jung war von Haus aus Katholik; nun trat er - offenbar innerlich schon lange dem Protestantismus zugeneigt - in die evangelische Kirche über. Fries und Schleiermacher, der Philosoph wie der Theologe, stimmten darin überein, daß sie einen unüberbrückbaren Graben zwischen Vernunft und Glauben, zwischen Christentum und Wissenschaft nicht gelten ließen: Ratio und Fides schließen sich nicht aus, sie ergänzen und erleuchten sich vielmehr gegenseitig. Diese Überzeugung ist auch für Karl Gustav Jung Zeit seines Lebens beglückende Wahrheit geblieben.

In Berlin blühte seit der Gründung der Universität (1810) das geistige Leben wie nie zuvor. Auch äußerlich entwickelten sich die Dinge zunächst für Jung günstig: er wurde Assistent des Chirurgen und Augenarztes Johann Nepomuk Rust (1775-1840) an der Charité und bekam dazu einen Lehrauftrag für Chemie an der königlich-preußischen Kriegsschule. Da wurde im Frühjahr 1819 August von Kotzebue (geb.1761), Dichter, aber zugleich russischer Staatsrat und daher für viele der Inbegriff eines reaktionären und servilen Geistes, von dem Theologiestudenten Sand ermordet. Dieses Ereignis war das Signal für scharfe Polizei- 
maßnahmen gegen die «Demagogen», d. h. offenkundig freiheitlich gesinnte Leute, vor allem unter den Akademikern, in allen deutschen Staaten: Burschenschaften und Turnvereine wurden unterdrückt, die Zensur verschärft; Fries in Jena wurde seiner Professur enthoben; der Buchhändler Reimer in Berlin mußte sich das Durchmustern seiner gesamten geschäftlichen und privaten Briefschaften gefallen lassen; eine ganze Reihe junger Akademiker wurde verhaftet, darunter Karl Gustav Jung am 7. Juli 1819. In Jungs Besitz fand die Polizei ein Geschenk des Mörders Sand: einen Hammer für geologische Untersuchungen! Dieses Instrument mußte seinen Eigentümer offenbar als besonders staatsgefährlich erscheinen lassen. Jedenfalls wurde Jung volle dreizehn Monate in der Berliner «Hausvogtei» in Haft behalten, dann ohne jedes Gerichtsurteil entlassen und aus Preußen ausgewiesen.

Damit war seine so hoffnungsvoll begonnene Berliner Laufbahn jäh beendet. Aber auch die deutschen Hochschulen außerhalb Preußens boten dem durch seine unverschuldete Haft zum «Demagogen» gestempelten jungen Gelehrten nun keine Möglichkeit des Fortkommens mehr, und allein der Ausübung der ärztlichen Praxis, abseits der Universität, mochte Jung offenbar nicht leben. Um aus seiner trüben Lage das Beste zu machen, ging er im Februar $1821^{3}$ nach Paris, dem glänzendsten medizinischen und naturwissenschaftlichen Zentrum des kontinentalen Europa: dort konnte er wenigstens unbehelligt von polizeilicher Schnüffelei sich wissenschaftlich fortbilden.

In Paris hatte Karl Gustav Jung das Glück, Alexander von Humboldt (1769 bis 1859) zu begegnen. Jahrzehnte später, am 6. Mai 1859, schrieb er zurückblickend in sein Tagebuch ${ }^{4}$ :

«Heute ist, nach eingelaufenem Bericht, Alexander von Humboldt nach einem kurzen Krankenlager gestorben ... Was er aber mir, dem unwürdigen Schreiber dieser Zeilen, gewesen, wird in mir leben, solange ich selbst lebe. Liebe und Dank dem großen, liebenden, edlen Manne. „Er wollte gut machen, was sein Gouvernement mir durch eine Reihe von Ungerechtigkeiten geschadet habe." Mit diesen Worten bot er mir, dem Verbannten, Verstoßenen, Verlassenen die Hand in Paris. Der Unvergeßliche, Gütige hat Wort gehalten. Ich aber auch!»

Über die Umstände der Begegnung sind wir nur unzulänglich unterrichtet. Der Psychiater Hermann Reimer (geb. 1825), Sohn des oben erwähnten Berliner Buchhändlers und Jungs Schwiegersohn, hat davon eine reichlich phantasievolle Darstellung gegeben ${ }^{5}$. Danach wäre Jung bei einem von dem Chirurgen Dupuytren gegebenen Festessen von einem würdigen Herrn mittleren Alters angesprochen und aufgefordert worden, 


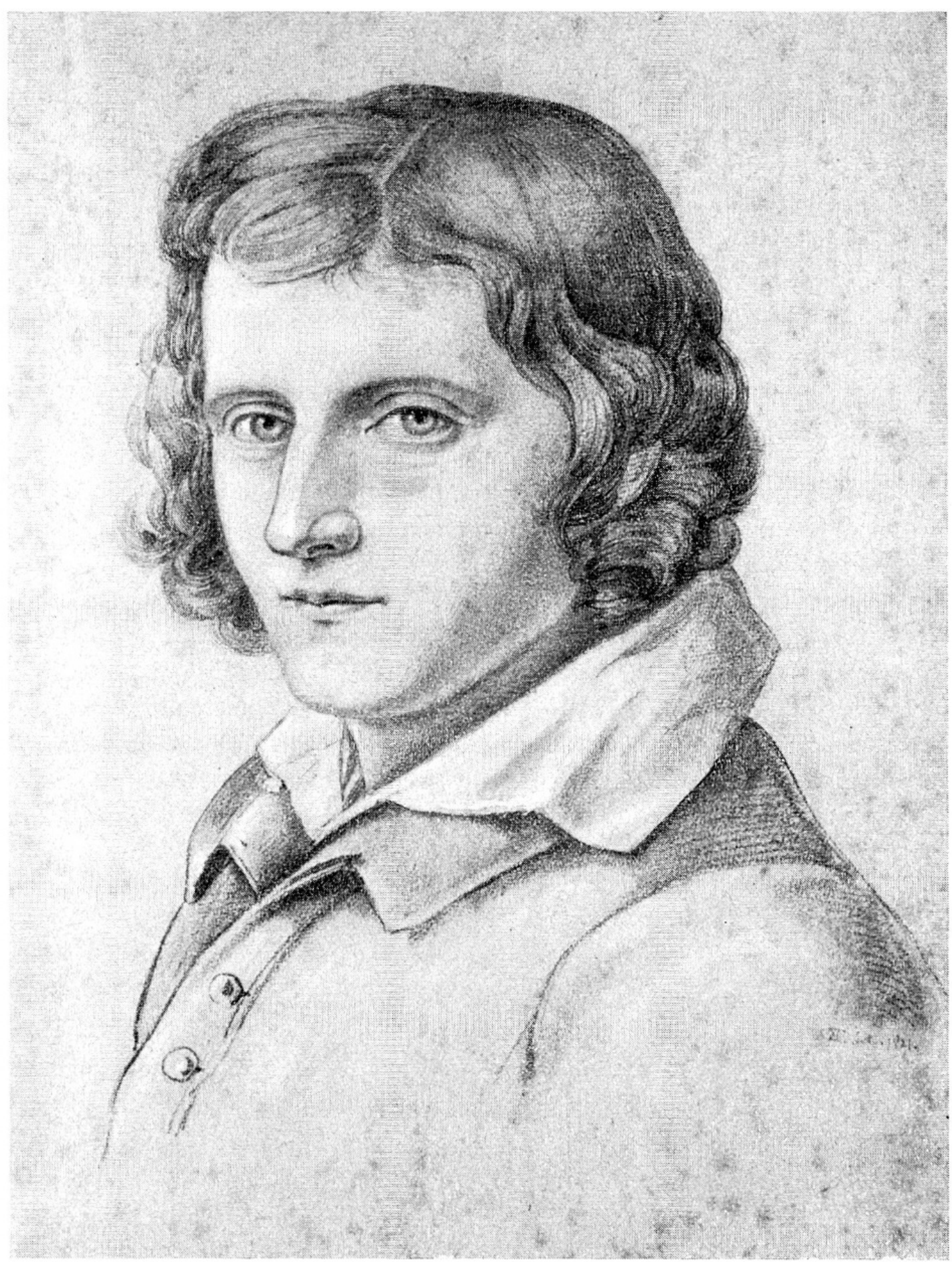

Karl Gustav Jung. Zeichnung des besonders als Landschaftsmaler bekannten Ernst Fries (1801-1833) von Heidelberg. Privatbesitz. - Die Datierung 'Februar 1821' erlaubt den Schluß, daß das Bild entstanden ist, als Jung nach seiner Ausweisung aus Preußen in seiner Heimat (Mannheim) weilte, unmittelbar bevor er sich nach Paris begab. 
«ihm nach Tisch in seine Wohnung zu folgen, weil er ihm einen Vorschlag zu machen habe. Blindlings folgte Jung dieser Aufforderung, und kam erst wieder zur Besinnung, als sein Gönner in seinem Arbeitszimmer ihm sagte, es handle sich um eine Professur für Anatomie und Chirurgie an der neugegründeten (!) Universität zu Basel, falls er dazu Lust hätte. Jetzt konnte er nicht mehr an sich halten, sondern raffte sich zu der Frage auf, wem er so viel Güte und dieses Glück zu verdanken habe. Darauf jener: ,Der Name tut nichts zur Sache, ich heiße Alexander von Humboldt.'» H. Reimer fügt bei: «Die Kenntnis von Jungs Erlebnissen konnte A.v.H. daher haben, daß er in literarischer Beziehung häufig mit meinem Vater zu tun hatte, aber auch von seinem Bruder Wilhelm, der mißmutig 1819 seinen Ministerposten verließ.»

Die Wirklichkeit sah weniger glänzend aus. Karl Gustav Jung lebte über ein Jahr lang von rund 600 Gulden, die ihm der Advokat Dr. Saur in Frankental (Pfalz) geliehen hatte ${ }^{6}$. «In Paris war er am Verhungern, als Alexander v. Humboldt ihn zufällig auf einer Bank entdeckte», berichtete sein Enkel C.G.Jung aufgrund der feststehenden Familientradition (vgl. den im Anhang publizierten Brief). Auch war Humboldt bei all seinem Ansehen doch nicht ein Magier, der eine schweizerische Professur seinem Schützling einfach so zuspielen konnte. Und ehe Jung sich nach Basel wandte, bewarb er sich, ebenfalls mit Humboldts Unterstützung, um einen Lehrstuhl an der Berner Akademie, die 1805 reorganisiert und zu einer eigentlichen Hochschule mit vier Fakultäten erweitert worden war ${ }^{7}$. Am 26. November 1821 wandte er sich folgendermaßen an den Präsidenten der akademischen Curatel, Abraham Friedrich von Mutach (1765-1831) ${ }^{8}$, in Bern:

«Hochwohlgeborener, insbesonders hochzuverehrender Herr Rathsherr und Kanzler.

Indem ich mir die Freiheit nehme Euer Hochwohlgeboren beiliegendes Schreiben vorzulegen, wage ich um die Verleihung der vakanten Lehrstelle der Chemie bei der Akademie in Bern zu bitten. Ich habe mich mehre Jahre hindurch unter der Anleitung Gmelin's in Heidelberg anhaltend mit Chemie beschäftigt; bin späterhin selbst als Lehrer aufgetreten, und habe mich vor ungefähr sechs Monaten hierherbegeben, um die persönliche Bekanntschaft derjenigen Gelehrten zu machen, denen der chemische Theil der Naturwissenschaft seine vollendete Stellung verdankt. Aufgeregt durch den lebhaften Wunsch für eine Akademie zu arbeiten, die Euer Hochwohlgeboren bekanntem Interessen für die Wissenschaft ihre Blüthe verdankt, und fest in dem Bewußt- 
sein meiner in der Chemie gewonnenen nöthigen Ausbildung glaubte ich an Euer Hochwohlgeboren meine Bitte richten zu dürfen.

Mit tiefster Hochachtung hat zu verharren die Ehre

Euer Hochwohlgeboren

Paris den 26 ten November hôtel de Rouen dans la cour du commerce près 1821 de la rue St. André des arts.»

Das in Jungs Brief erwähnte Empfehlungsschreiben stammte, wie aus dem Protokoll der Curatel hervorgeht, von Alexander von Humboldt; es ist im Berner Staatsarchiv nicht mehr vorhanden.

Jungs Bewerbung ging am 17. Dezember 1821 bei der Curatel ein; am Mittwoch, den 26. Dezember, faßte die Aufsichtsbehörde folgenden Beschluß ${ }^{9}$ :

«Vortrag an MeGdHh. die Räthe

Auf die Ausschreibung, des durch den Tod des Herrn Professors Beck, an hiesiger Akademie erledigten Lehrstuhls der Chemie, wozu die Akademische Curatel von Euer Gnaden unterm $22^{\text {t. }}$ Oktober ermächtiget worden war, hatte sich dafür gemeldet:

Hr. Dr. Carl Brunner, Apotheker allhier.

Hr. Dr. Giesberger, Arzt bey dem Regiment v. Jen[n]er in Königl. Holländischen Diensten.

Hr. Dr. Jung gegenwärtig in Paris.

... der letztere dann, Herr Dr.Jung, unterstützte seine Bewerbung mit einer sehr günstig lautenden Empfehlung des in der litterarischen Welt rühmlichst bekannten Herrn Alexanders von Humboldt.

Wegen der Entfernung dieser beiden Bewerber [Giesberger und Jung], war einzig Herr Dr. Brunner im Fall, die für das erledigte Catheder aufgegebenen Proben zu bestehen. Diese Proben enthielten zwey Aufgaben, eine theoretische, wozu eine öffentlich vorzutragende Abhandlung über die neuere Geschichte der Chemie mit Aushebung und Beurtheilung der verschiedenen Systeme bezeichnet wurde, und zweytens eine praktische Aufgabe, wozu man die chemische Zerlegung des chromsauren Eisens der Bern-hills bey Baltimore ausgewählt hatte. -

Diese beyden Proben hat nun Herr Brunner laut dem Zeugniß des Examinatoren Collegiums ... auf die befriedigendste Weise abgelegt ...»

Eine Woche später, am Berchtoldstag, dem 2. Januar 1822, legte der neugewählte Professor Karl Brunner (1796-1867) bereits sein Amtsgelübde in die Hand des 
Curatel-Präsidenten ab und erhielt dafür sein «Patent». Er versah sein Lehramt als Professor der Chemie und Pharmazie 40 Jahre lang und galt daneben als tüchtiger Landschaftsmaler. Ohne Zögern zogen die Berner Behörden also den soliden Mitbürger dem vielleicht brillanteren, aber unbekannten Schützling Humboldts vor.

Ohne sich darum beworben zu haben, stand Karl Gustav Jung im Frühjahr 1822 in Freiburg im Breisgau als Anwärter auf den Lehrstuhl der Chemie zur Diskussion $^{10}$. Er selber hatte aber bereits ein anderes Ziel ins Auge gefaßt: die Basler Professur für Anatomie, Chirurgie und Geburtshilfe. Sein Mißerfolg in Bern hat wohl dazu beigetragen, daß er nun in Basel sehr aktiv und zielbewußt vorging. Seinem ersten (im Basler Staatsarchiv nicht vorhandenen) Bewerbungsschreiben an den Bürgermeister Johann Heinrich Wieland (1758-1838) legte er gleich vier gewichtige Empfehlungen bei. Drei stammten von seinen akademischen Lehrern, dem Pariser Chirurgen und Anatomen Gilbert Breschet (1784 bis 1845), dem Heidelberger Chemiker Leopold Gmelin (1788-1853) und dem Geburtshelfer Franz Karl Naegele (1778-1851), ebenfalls in Heidelberg. Aber das Glanzstück war selbstverständlich der Brief Alexander von Humboldts an Bürgermeister Wieland ${ }^{11}$ :

«Monsieur,

Je m'adresse avec confiance au magistrat que les vœux libres de ses concitoyens ont placé dans une sphère élevée et qui se plaît à employer son influence à faire prospérer les institutions utiles, à conserver à sa noble patrie la gloire littéraire qu'elle s'est acquise dans les siècles passés. J'ose Vous recommander, Monsieur, un savant sur lequel Vous daignerez fixer votre attention et qui m'intéresse vivement et par la variété et l'étendue de ses connaissances et par les qualités morales qui n'accompagnent pas toujours le savoir et les talens littéraires. Mr. le Docteur Joung serait sans doute un ornement de Votre Université : il avait déjà acquis en Allemagne, sous de grands maîtres, l'habitude des opérations chirurgicales. Ses connaissances distinguées l'ont fait marquer ici, dans un pays où l'on n'est pas toujours équitable pour les habitans de la rive droite du Rhin et où la Chirurgie compte encore dans les hôpitaux et les chaires publiques, les hommes les plus illustres. Un long séjour à Paris a été très utile à Mr. Joung. Il a professé une autre science, la Chimie, avec un grand succès à Berlin et cette habitude de professer lui procurera de nouveaux avantages dans Votre Université. Il me serait doux d'apprendre, Monsieur, que mon témoignage aye pu lui être favorable auprès de Vous. Tout en Vous suppliant d'excuser l'indiscrétion d'un homme de lettres qui désire être utile à un 


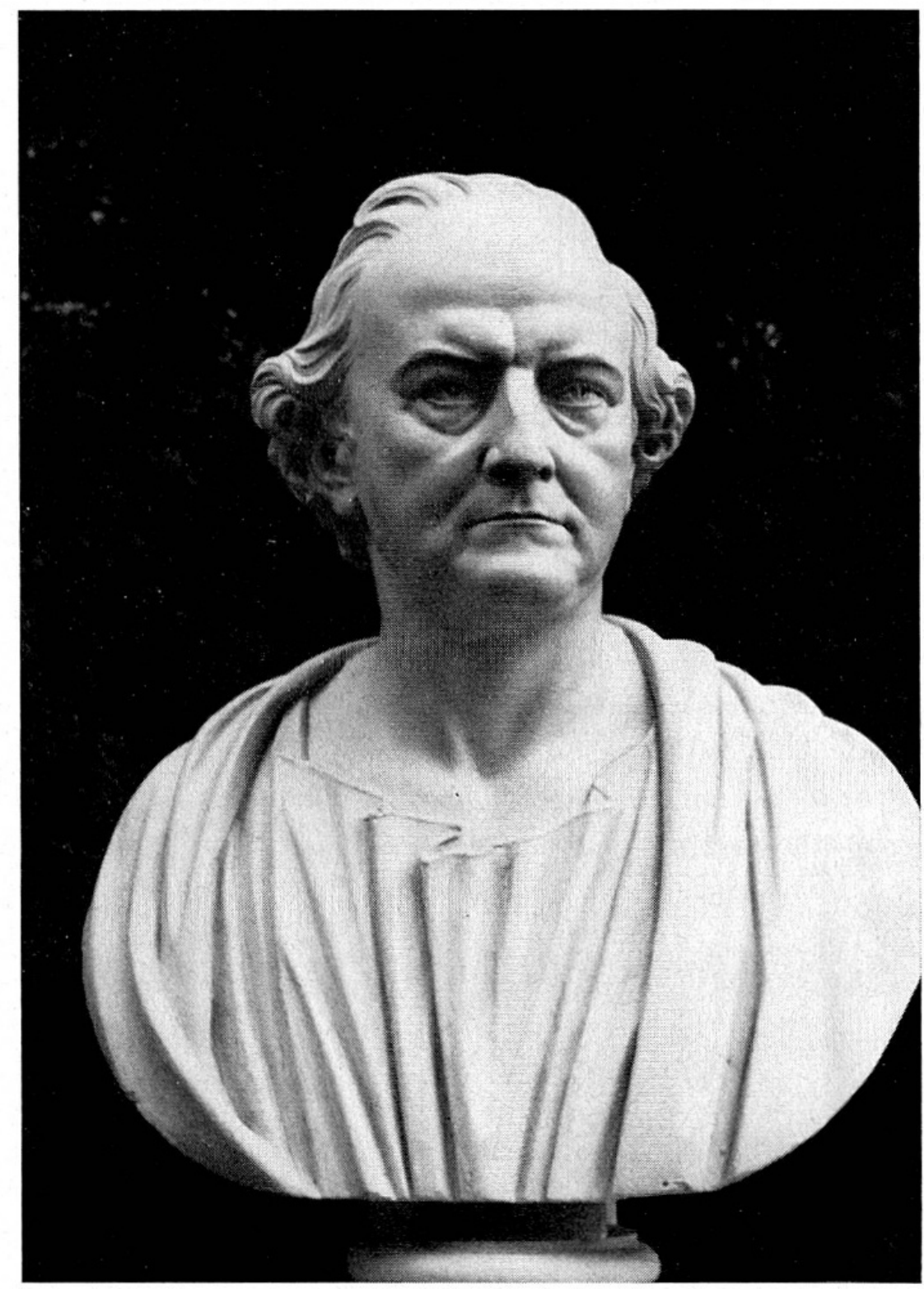

Karl Gustav Jung in späteren Jahren

Gipsbüste im Medizinhistorischen Institut der Universität Zürich Photographie: Heinz Balmer 
jeune savant bien digne de Votre haute protection, je Vous supplie d'agréer l'hommage de la considération respectueuse avec laquelle j'ai l'honneur d'être, Monsieur,

Paris quai de l'Ecole 26 Votre très-humble et très-obéissant serviteur ce 5 Janv. 1822 Al. de Humboldt de la Société d'histoire naturelle helvétique.»

Wieland antwortete ausweichend ${ }^{12}$ :

«Monsieur le Baron,

Si la Lettre dont Vous m'avez honoré le 5 Janv passé, m'a causé un plaisir bien sensible en me prouvant l'intérêt que vous voules bien vouer à notre Suisse, il me peine vivement de ne pouvoir, malgré mes délais, répondre d'une façon satisfaisante à la recommendation que vous m'avez adressé. La faculté de Médecine de notre université se trouve entièrement dépourvue de Professeurs et manque par une suite très naturelle d'Etudians. Dans cet étât de chose la Curatèle ne peut pourvoir à une des trois places et se voit obligé de soumettre à la décision du Conseil d'Education la question génerale: si ces places resteront encore quelque tems suspendues, ou si on pourroit profiter de l'exellente occasion que Vous nous fournissés de procurer a notre université et à notre public un Sujet aussi distingué que Monsieur Joung.

Dès que ma proposition sera agréé je ne manquerois pas de Vous en instruire. Veuilles en attendant agréer l'assurance de ma considération respectueuse avec laquelle j'ai l'honneur d'être

Monsieur le Baron

Basle ce 5 Mars

1822
Votre très humble et très obeïssant serviteur.

Wieland, Bourgmestre

et Chancelier de l'université de Basle.»

Offenbar hatte Karl Gustav Jung dieses Schreiben vor sich, als er postwendend antwortete ${ }^{13}$ :

«Euer Excellenz

Gütiges Schreiben habe ich erhalten und nehme mir sofort die Freiheit meinen tiefsten Dank für dasselbe auszusprechen. Obschon mich die Verzögerung der Erfüllung meiner schönen Hoffnungen betrübt, so fühle ich mich doch mächtig durch das Bewußtsein gehoben von Euer Excellenz begünstig zu sein. In der Überzeugung als Wundarzt mit Vortheil für meine Umgebung wirken zu können und besonders durch die Gewißheit von Eurer Excellenz, bei der 
Besetzung der Lehrstelle der Wundarzneikunde berücksichtigt zu werden, geleitet, habe ich mich entschlossen Paris an einem der folgenden Tage zu verlassen und mich nach Basel zu begeben. Im Vertrauen auf Euer Excellenz Güte werde ich ergebenst um Rath bitten, da ich wünsche, die Erlaubnis zu erhalten, zunächst als ausübender Wundarzt in Basel auftreten zu dürfen. Ich glaube daß Euer Excellenz allesvermögende Unterstützung für mich noch bedeutender werden müßte, wenn Hochdieselben mich in meinem wissenschaftlichen Wirken unmittelbar zu beurtheilen Gelegenheit haben werden. Glauben Eure Excellenz nicht daß Eitelkeit mich diese Worte sagen läßt, sondern daß sie aus der festen Überzeugung meiner nötigen Ausbildung entspringen, der Überzeugung, die mich es schon hatte wagen lassen, mit den Empfehlungen berühmter Männer ausgerüstet, an Eure Excellenz zu schreiben.

Mit ganz besonderer Hochachtung unterzeichnet

Eurer Excellenz gehorsamst-ergebenster Diener

Paris den 11ten März 1822 Dr. Jung»

Schon am 20. März erschien Jung in Basel und stellte das Gesuch, im bevorstehenden Semester als Privatdozent über Geburtshilfe, chirurgische Arzneimittellehre und Verbandlehre öffentliche Vorlesungen halten zu dürfen. Am gleichen Tag bewilligte die Curatel der Universität sein Gesuch ${ }^{13}$.

Der heutige Leser der zitierten Dokumente staunt über Jungs Vielseitigkeit und Selbstsicherheit: derselbe Mann, der sich eben noch den Bernern als kompetenten Chemiker empfahl, brennt nun darauf, in Basel als Chirurg zu praktizieren und Geburtshilfe zu lehren!

Am 20. März 1822 wurde die vakante Professur im Basler Kantonsblatt ausgeschrieben. Neben Jung meldeten sich Johann Baptist Sartory von Rappenstein (1787-1865), praktischer Arzt in Andeer in Graubünden, und Johann Jakob Mieg (1794-1870) aus Basel. Jakob Mieg hatte schon 1819 als Privatdozent Chirurgie gelesen. Später (1830) wurde er zum Ordinarius für Chirurgie und Geburtshilfe ernannt, während Jung dannzumal die Anatomie behielt und die innere Medizin übernahm. Jetzt, im Sommer 1822, suchten sich die Basler Behörden aber wirklich den besten der drei Kandidaten aus: im Gegensatz zu den Bernern gaben sie dem in Heidelberg, Berlin und Paris ausgebildeten und somit wissenschaftlich überlegenen Kandidaten den Vorzug. Ende Juni 1824 wurde Karl Gustav Jung zum ordentlichen Professor der Chirurgie, Anatomie und Entbindungskunst an der Universität Basel gewählt ${ }^{14}$. Seine Schulden bei Dr. Saur drückten ihn trotzdem noch jahrelang. 
Noch ein Wort zu Alexander von Humboldts Rolle in der Lebensgeschichte Karl Gustav Jungs. Nicht nur nahm er sich uneigennützig und nachhaltig um den hilfsbedürftigen Flüchtling an, dessen Befähigung zur Professur er richtig einschätzte; er zeigte sich auch außerordentlich gut über die Verhältnisse an den schweizerischen Hochschulen unterrichtet. Daß Basel einen Anatomie- und Chirurgieprofessor brauchte, wußte er ungefähr ein Vierteljahr vor der amtlichen Ausschreibung. Die Persönlichkeit, die ihn auf dem laufenden hielt, dürfte Philipp Albert Stapfer (1766-1840) gewesen sein, der der Helvetischen Republik als Minister der Künste und Wissenschaften und als Gesandter in Paris gedient hatte, nachher als Privatgelehrter in Frankreich blieb und mit Humboldt befreundet war. Dieser Freundschaft ist es somit zu danken, daß Karl Gustav Jung in die Schweiz kam. Das Vertrauen, das das liberale Basel ihm entgegenbrachte, hat er seinerseits glänzend gerechtfertigt.

\section{Anhang}

Die Archivstudien, auf denen der vorangehende Aufsatz beruht, liegen weit zurück. Am 26. September 1954 habe ich einen Teil der Ergebnisse im Sonntagsblatt der inzwischen leider untergegangenen «Basler Nachrichten» zum erstenmal veröffentlicht unter dem Titel « Wie Karl Gustav Jung Basler Professor wurde». Prof. Dr. C. G. Jung in Küsnacht (Zürich) schrieb mir darauf am 27. Oktober 1954:

«Sehr geehrter Herr!

Entschuldigen Sie bestens die Verspätung meiner Antwort. Ich möchte es aber doch nicht versäumen, Ihnen für die freundliche Zusendung Ihres Artikels über meinen Großvater zu danken.

Das Andenken an Sand besteht nicht in einem «Beil», sondern in einem geologischen Hammer.

Unter meinem Großvater wurde auch die neue Irrenanstalt hinter dem Spital bei der Predigerkirche gebaut.

In Paris war er am verhungern, als Alexander v. Humboldt ihn zufällig auf einer Bank entdeckte. Ich besitze Bürgermeister Wielands Brief an Humboldt und das Taufzeugnis, das Schleiermacher für meinen Großvater ausgestellt hat. Auch besitze ich noch sein schwarz-rot-goldenes Band, das er als Student trug. Als Student in Heidelberg hielt er sich statt eines Hundes ein zahmes Schwein, wodurch er allgemeines Aufsehen erregte.

Mit vorzüglicher Hochachtung

Ihr ergebener

C. G. Jung. » 
Im Frühjahr 1955 gewährte mir C.G.Jung Zugang zu seinem Privatarchiv; einiges daraus ist in der vorliegenden Arbeit, mit freundlicher Bewilligung von Herrn dipl. Arch. Franz Jung in Küsnacht, ebenfalls berücksichtigt. Die Biographie, die er verdienen würde, hat Karl Gustav Jung der Ältere jedoch noch nicht erhalten.

\section{Anmerkungen}

1 H. Balmer, Alexander von Humboldt und Frankreich, Gesnerus 33 (1976), S. 235-251. Zitat von S. 247.

2 Chronik von Goethes Leben (Insel-Verlag), o. O.1949, S. 57-61.

3 Vgl. Anm. 6.

${ }^{4}$ E. Jung, Aus den Tagebüchern meines Vaters, Privatdruck Basel 1910, S. 224.

5 H. Reimer, Georg Andreas Reimer. Erinnerungen aus seinem Leben. Berlin 1900.

${ }_{6}$ Privatarchiv C.G.Jung, Nr. 72, Rückzahlungsverpflichtung vom 20. Sept. 1829.

7 In einem Zusatz schreibt K.G. Jung von den «614 Gulden, mit denen ich in Paris 13 Monate gelebt habe. » Da er Mitte März 1822 Paris verließ, muß er im Februar 1821 dort eingetroffen sein. F. Haag, Die hohen Schulen zu Bern ... von 1528-1834, Bern 1903.

8 Staatsarchiv Bern, B III 955.

9 Manual der akadem. Curatel, Staatsarchiv Bern, B III 915.

10 E.Th.Nauck, Zur Vorgeschichte der naturwissenschaftlich-mathematischen Fakultät der Albert-Ludwigs-Universität Freiburg i.B., Freiburg 1954, S. 42.

11 Staatsarchiv Basel, AA 6. Die Akzente wurden entsprechend den heutigen Regeln gesetzt.

12 Privatarchiv C.G. Jung, Nr. 86.

13 Staatsarchiv Basel, AA 6.

14 Werner Kolb, Geschichte des anatomischen Unterrichtes an der Universität zu Basel 1460 bis 1900, Basel 1951, S.104. - Kolb gibt auf S. 104-114 eine sorgfältige Darstellung von Jungs anatomischer Lehrtätigkeit in Basel.

\section{Weitere Literatur}

E. Bonjour, Die Universität Basel ... 1460-1960, Basel 1960.

A.Burckhardt, Geschichte der medizinischen Fakultät zu Basel 1460-1900, Basel 1917.

H.M.Koelbing, Carl Liebermeister (1833-1901), der erste Chefarzt der Basler medizinischen Universitätsklinik, Gesnerus 26 (1969), S. 233-248.

A.Staehelin, Die Universität Basel am Anfang des 19. Jahrhunderts, Basler Zeitschrift 52 (1953), S.119-137. 


\section{Summary}

Karl Gustav Jung, M.D. (1794-1864), the grandfather of C. G. Jung the psychologist, came to Paris, in 1821, as a liberal refugee from Germany. Guided by Alexander von Humboldt, he wrote, while still in Paris, applications first for the chair of chemistry at the Academy of Berne, then for the chair of anatomy, surgery and midwifery at the venerable University of Basle. The author describes Jung's way to Switzerland on the basis of archive documents and letters. With Jung's appointment in 1822, the languishing Faculty of Medicine in Basle arose to new life.

Prof.Dr.med. Huldrych M. Koelbing

Medizinhistorisches Institut

Rämistraße 71

8006 Zürich 